\title{
Analysis of mRNA-IncRNA and mRNA-IncRNA- Pathway co-expression networks based on WGCNA in developing pediatric sepsis
}

\section{Xiaojuan Zhang}

Zhengzhou University First Affiliated Hospital

\section{Yuqing Cui}

Zhengzhou University First Affiliated Hospital

\section{Xianfei Ding}

Zhengzhou University First Affiliated Hospital

Shaohua Liu

Zhengzhou University First Affiliated Hospital

\section{Bing Han}

Zhengzhou University First Affiliated Hospital

\section{Xiaoguang Duan}

Zhengzhou University First Affiliated Hospital

\section{Haibo Zhang}

University of Toronto

Tongwen Sun ( $\nabla$ suntongwen@163.com )

Zhengzhou University First Affiliated Hospital

\section{Research article}

Keywords: pediatric sepsis, mRNA, IncRNA, diagnostic markers, WGCNA

Posted Date: August 13th, 2020

DOI: https://doi.org/10.21203/rs.3.rs-54798/v1

License: (c) (1) This work is licensed under a Creative Commons Attribution 4.0 International License. Read Full License

Version of Record: A version of this preprint was published at Bioengineered on January 1 st, 2021. See the published version at https://doi.org/10.1080/21655979.2021.1908029. 


\section{Abstract}

Background: Pediatric sepsis is a great threat in death worldwide. However, the pathogenesis has not been clearly understood until now in sepsis.

Methods: This study identified differentially expressed mRNA (DEMs) and IncRNAs (DELs) based on Gene Expression Omnibus (GEO) database. And the weighted gene co-expression network analysis (WGCNA) was performed to explore co-expression modules associated with pediatric sepsis. Then Gene Ontology (GO), KEGG (Kyoto Encyclopedia of Genes and Genomes) pathway, DEMs-DELs and DEMs-DELs-Pathway co-expression network analysis was conducted in selected significant module.

Results: A total of 1941 DEMs and 225 DELs were used to conduct WGCNA. And the turquoise module was selected as the significant module that was associated with particular traits. The DEMs functions associated with many vital processes were also shown by GO and KEGG pathway analysis in the turquoise module. Finally, 15 DEMs and 4 DELs (GSEC, NONHSAT160878.1, XR_926068.1 and RARAAS1) were selected as candidate biomarkers in DEMs-DELs-Pathway co-expression network.

Conclusions: Our study identified 15 DEMs and 4 DELs as diagnostic markers, which could also provide more directions to study molecular mechanism of pediatric sepsis.

\section{Background}

There are about 1.2 million childhood sepsis cases to be diagnosed globally per year[1]. Unfortunately, mortality was reported to be as high as $25 \%-50 \%$ for children hospitalized for sepsis[2]. The definition of sepsis has been revised repeatedly, and the latest adult sepsis definition cannot be rigidly applied to children, so the early diagnosis and appropriate managements essential to improve clinical outcomes for children at risk for sepsis[3]. Therefore, understanding the potential molecular mechanism underlying sepsis is critical to identify early diagnostic biomarkers and find effective drugs.

Long noncoding RNAs (IncRNAs) are a type of non-protein transcripts that exceed 200 nucleotides in length[4]. LncRNAs may be involved in mRNA splicing and maturation, mRNA transport or localization, mRNAs stabilization[5]. There was increasing evidences that IncRNAs had critical regulatory effects on the pathophysiology and organ dysfunction in sepsis[6]. For instance, IncRNA NEAT1 played a vital role in inducing acute kidney injury by modulating NF-KB pathway. LncRNA ENST00000504301.1 and ENST00000452391.1 were reported had differential expression between sepsis survivors and nonsurvivors[7]. But these reports only showed the role of one or two IncRNA molecules in sepsis. The differentially expressed mRNA (DEMs) and IncRNAs (DELs) regulatory network may provide a better understanding for us of mRNA and IncRNA biological function. Co-expression analyses of DEMs-DELs reflect the potential function of IncRNAs. However, a previous study[8] only analyzed the function of DEMs, no study performed weighted gene co-expression network analysis (WGCNA) to systematically construct DEMs-DELs and DEMs-DELs-Pathway co-expression networks concerning pediatric sepsis. Therefore, we made a differential expression analysis followed by WGCNA to explore modules associated 
with pediatric sepsis and investigate the interactive relationships between DEMs and DELs by DEMs-DELs and DEMs-DELs-Pathway co-expression networks.

In this study, 1941 DEMs and 225 DELs were identified based on RNA-seq datasets of the whole spectrum of systemic inflammatory response syndrome (SIRS), sepsis, septic shock, SIRS resolved and control group at day 1 and day 3 from Gene Expression Omnibus (GEO) database. Then WGCNA and module-trait relationships were conducted to select important modules. Subsequently, function and pathway enrichment analysis of important module were conducted by Gene Ontology (GO) and Kyoto Encyclopedia of Genes and Genomes (KEGG). DEMs-DELs and DEMs-DELs-Pathway networks also were constructed in significant modules. Thus, this comprehensive analysis might provide new insights into DEMs-DELs and DEMs-DELs-Pathway co-expression roles in pediatric sepsis. Selective DEMs and DELs may become diagnostic and therapeutic targets associated with pediatric sepsis.

\section{Methods}

\section{Data Collection}

Expression dataset of sepsis (GSE13904) were downloaded from the GEO database (https://www.ncbi.nlm.nih.gov/geo/query/acc.cgi?acc=GSE13904) and then collated into standardized raw data for subsequent analysis. The mRNAs and IncRNAs expression data of 227 children included 18 control samples, 32 sepsis samples, 67 septic shock samples and 22 SIRS samples on day 1 . The day 3 samples consisted of 20 sepsis patients, 39 septic shock patients, 24 patients meeting SIRS resolved and patients had paired day 1 and day 3 .

\section{DEMs and DELs screening}

Limma package in R was used to find the DEMs and DELs between the four related symptoms (Sepsis,Septic Shock $\square$ SIRS and SIRS resolved) and control samples at two different time points (day1, day3). The differential screening parameter was set as: $P<0.05$ \& false discovery rate (FDR) $<0.05$ \& fold change $(F C)>2$ to obtain the DEMs and DELs for WGCNA analysis.

\section{Construction of WGCNA network}

1941 DEMs and 225 DELs were performed to build the co-expression modules by WGCNA. The cluster analysis was shown that all the samples were in the clusters, so it is not necessary to remove the outlier. The soft threshold power $\beta$ was set at 16 , which was more consistent with scale-free networks and had a more biological significance[10]. The independence had a high degree and the average connectivity had lower level when the power value was equal to 16 (Fig. 1a). Therefore, $\beta=16$ was used to engender a hierarchical clustering tree. These DEMs and DELs with similar expression patterns were put into modules through average link clustering (Fig. 1b). 11 modules (turquoise, grey, blue, brown, yellow, green, red, 
black, pink, magenta and purple) were recognized and exhibited with different colors totally, and the number of DEMs and DELs in these modules were shown in (Table 1).

Table 1

The number of DEMs and DELs in the 11 modules.

\begin{tabular}{|llll|}
\hline Module & All numbers & DEMs & DELs \\
\hline turquoise & 494 & 468 & 26 \\
\hline grey & 482 & 410 & 72 \\
\hline blue & 410 & 370 & 40 \\
\hline brown & 380 & 352 & 28 \\
yellow & 81 & 71 & 10 \\
\hline green & 72 & 70 & 2 \\
\hline red & 72 & 71 & 1 \\
black & 53 & 49 & 4 \\
pink & 52 & 50 & 2 \\
\hline magenta & 48 & 8 & 40 \\
\hline purple & 22 & & 22 \\
\hline
\end{tabular}

\section{Module-trait relationships analysis}

The correlation between co-expression modules and clinical traits was estimated base on the phenotypic information of pediatric sepsis, septic shock, SIRS, SIRS resolved at day 1 and day3 and control group. The significant co-expression module highly related to traits was identified. Module-trait relationships were computed by Pearson's correlation tests, and $P<0.05$ was defined significant correlation.

\section{Functional enrichment analysis of significant co-expression modules}

DAVID (http://david.abcc.ncifcrf.gov/) was a database for annotation, visualization and integrated discovery. GO and KEGG pathway analysis of DEMs were conducted by DAVID. The ontology contained three classes: biological process (BP), molecular function (MF) and cellular component (CC). Fisher exact test and multiple comparison test were used to calculate significance level ( $p$-value), and $p$-value was corrected by FDR. Thus, the function and pathway of DEMs were identified in the significant module. 


\section{Construction of DEMs-DELs network and DEMs-DELs- Pathway}

The DEMs-DELs co-expression networks of significant module were carried out to explore the association between DEMs and DELs. According to DEMs-DELs network and the significant pathways involved in the regulation of DEMs, the network of DEMs-DELs-Pathway was constructed. Significant correlation pairs were applied to build the network in accordance with Pearson correlation coefficients. And the differential co-expression network chats were visualized and analyzed using Cytoscape software (Version 3.5.1).

\section{Statistical analysis}

Statistical analysis were conducted by R software 3.4.0 and SPSS 19.0. Fisher exact and multiple comparison test was used to find differences between traits. $p<0.05$ was supposed to be significant.

\section{Results}

\section{Identification of DEMs and DELs}

Our analysis identified 1110 DEMs and 111 DELs in the comparison groups control vs. pediatric sepsis day1, 435 DEMs and 37 DELs in the groups control vs pediatric sepsis day3, 1278 DEMs and 134 DELs in the groups control vs pediatric septic shock day1, 1565 DEMs and 172 DELs in the groups control vs pediatric septic shock day3, 760 DEMs and 77 DELs in the groups control vs pediatric SIRS day 1, 824 DEMs and 112 DELs in the groups control vs pediatric SIRS day3, 613 DEMs and 56 DELs in the groups control vs. pediatric SIRS resolved day3. Totally, we obtained 1941 DEMs including 927 (47.8\%) upregulated and 1014 (52.2\%) down-regulated mRNAs, and 225 DELs containing 132 (58.7\%) up-regulated and 93 (41.3\%) down-regulated IncRNAs between the four related symptoms (sepsis, septic shock, SIRS, SIRS resolved) and control group. And these DEMs and DELs were performed the hierarchical cluster analysis (Supplemental Figs. 1 and 2 (a-n)).

\section{Construction of WGCNA network}

1941 DEMs and 225 DELs were performed to build the co-expression modules by WGCNA. The cluster analysis was shown that all the samples were in the clusters, so it is not necessary to remove the outlier. The soft threshold power $\beta$ was set at 16 , which was more consistent with scale-free networks and had a more biological significance[10]. The independence had a high degree and the average connectivity had lower level when the power value was equal to 16 (Figure 1a). Therefore, $\beta=16$ was used to engender a hierarchical clustering tree. These DEMs and DELs with similar expression patterns were put into modules through average link clustering (Figure 1b). 11 modules (turquoise, grey, blue, brown, yellow, green, red, black, pink, magenta and purple) were recognized and exhibited with different colors totally, and the number of DEMs and DELs in these modules were shown in (Table 1). 


\section{Correlation analysis of module and traits}

The relationship between co-expression modules and clinic traits was identified in Fig. 2. Grey module included genes not belong to any module, and turquoise module was selected as significant module according to the max number of DEMs and DELs and most significant $p$ value. There were 468 DEMs and 26 DELs in the turquoise module.

\section{GO and KEGG pathway analysis}

The DEMs in the turquoise module were conducted GO analysis and KEGG pathway enrichment analysis. The results showed that neutrophil degranulation, inflammatory response, innate immune response and cytokine-mediated signaling pathway were enriched in BP, protein binding, protein homodimerization activity and ATP binding were mostly involved in MF, plasma membrane, extracellular exosome and cytosol were enriched in CC. KEGG pathway enrichment analysis suggested metabolic pathway, TNF signaling pathway and cytokine-cytokine receptor interaction were the most significant pathways (Fig. 3).

\section{Construction of the DEMs-DELs co-expression network}

DEMs-DELs co-expression networks were built to understand roles and functional mechanisms of DELs in turquoise module (Fig. 4). We found that 64 DEMs and 4 DELs were inter-regulated and one DEL was co-expressed with multiple DEMs. Besides, multiple DELs also had co-expression with one DEM, which suggested that a complicated regulatory association between DEMs and DELs existed in differential coexpression network.

\section{Construction of the DEMs-DELs-Pathway co-expression network}

According to the interaction network of DEMs-DELs and DEMs-Pathway, we obtained DEMs-DELsPathway networks (Fig. 5). In our study, we identified 15 DEMs and 4 DELs in the DEMs-DELs-Pathway network. And the 8 DEMs (MAPK14, ITGAM, HK3, ALOX5, CR1, HCK, NLRC4 and LTB4R) and 4 DELs (GSEC, NONHSAT160878.1, XR_926068.1 and RARA-AS1) had a significant association with pediatric sepsis. Mostly DELs-related DEMs could be attributed to functional categories involved in immune function, and metabolic pathway. We found that these pathways were markedly related to cytokinecytokine receptor interaction, complement and coagulation cascades, chemokine signaling pathway, and IL-17 signaling pathway.

\section{Discussion}


Early diagnosis of pediatric sepsis is a challenge but important to the effective treatment. And the mortality rate sharply increased when pediatric sepsis progressed severe sepsis and septic shock. Many studies displayed the essential role of genetic and epigenetic alterations as it related to sepsis diagnosis and treatment[11-14]. Therefore, molecular diagnosis can enhance the ability of rapid intervention and may decrease mortality rate.

In our study, 1941 DEMs and 225 DELs were identified and turquoise module significantly correlated with pediatric sepsis. The DEMs-DELs co-expression network construction of turquoise module provided an insight of correlation between DEMs and DELs. 64 DEMs and 4 DELs had interactions. And we also conducted DEMs-DELs-Pathway co-expression network and found 8 DEMs and 4 DELs had a significant association with pediatric sepsis. It revealed the signal pathway that DELs participates in, so as to predict the possible mechanism of DELs.

It had been reported that the MAPK14 (Mitogen-activated protein kinase 14) could activate MAPK signaling pathway to aggravate acute lung injury (ALI) in septic shock mice[15]. Moreover, the inhibition of MAPK14 could reduce the severity of ALI by inhibiting the activation of signaling pathway. In our study, MAPK14 co-expressed with GSEC, NONHSAT160878.1 and XR_926068.1 was enriched in MAPK signaling pathway, TNF signaling pathway, Toll-like receptor signaling pathway and IL-17 signaling pathway. Thence, we speculated that GSEC, NONHSAT160878.1 and XR_926068.1 might be involved in the progression of pediatric sepsis by regulating MAPK signaling pathway, TNF signaling pathway, Tolllike receptor signaling pathway and IL-17 signaling pathway.

ITGAM (integrin subunit alpha M), also known as CD11B, which was essential for the activation and migration of inflammatory cells. So it could mediate the inflammatory response. A previous study[16] had revealed that ITGAM mainly contributed to the progression of sepsis by promoting the nuclear and cytoplasmic translocation and activating release of HMGB1. And ITGAM blocking antibodies or inhibitors could protect mice from the lethality associated with LPS and microbial sepsis[17]. Studies also had found the expression of ITGAM in neutrophils and monocytes was highest in adult and neonatal sepsis, so its expression may be a promising method for the specific and sensitive diagnostic indicators[17, 19]. ITGAM co-expressed with GSEC, NONHSAT160878.1 and RARA-AS1 was enriched in phagosome pathway in our study. Therefore, we had the speculation that GSEC, NONHSAT160878.1 and RARA-AS1 might be involved in the progression of pediatric sepsis by regulating phagosome pathway.

Hexokinase 3 (HK3) phosphorylated glucose to produce glucose-6-phosphate and involved in the first step of glucose metabolism. The activity of HK3 increased rapidly after lipopolysaccharide (LPS) exposure, and was related to the increasing of pentose phosphate pathway activity. It suggested that the up-regulation of HK3 in sepsis was an important factor to interrupt energy production and cause AKI[20]. HK3 co-expressed with GSEC, NONHSAT160878.1 and RARA-AS1 was enriched in metabolic pathways, glycolysis or gluconeogenesis and insulin signaling pathway. Then, we inferred that GSEC, NONHSAT160878.1 and RARA-AS1 might be involved in the progression of pediatric sepsis by regulating metabolic pathways, glycolysis or gluconeogenesis and insulin signaling pathway. 
ALOX5, also known as 5-lipoxygenase (5-LO), was an important enzyme of producing leukotrienes (LTs) to cause lung damage in sepsis[21]. ALOX5 products could induce pulmonary inflammation and its inhibition attenuated sepsis-induced lung injury[22]. In this study, ALOX5 co-expressed with GSEC, NONHSAT160878.1, XR_926068.1 and RARA-AS1 was enriched in metabolic pathways, and Fc epsilon RI signaling pathway. These findings demonstrated that GSEC, NONHSAT160878.1, XR_926068.1 and RARA-AS1 might have a vital effect on the progression of pediatric sepsis by regulating metabolic pathways, and Fc epsilon RI signaling pathway.

The complement cascade was activated overwhelmingly and showed deleterious effects in sepsis. Some study reported erythrocyte CR1, a complement receptor 1, protected bacteria from leukocyte phagocytosis in human blood[23]. And CR1 monoclonal antibody could ameliorate sepsis-induced mortality during Staphylococcus aureus infection[24]. In our results, CR1 co-expressed with GSEC and NONHSAT160878.1 was enriched in complement and coagulation cascades. So GSEC and NONHSAT160878.1 might be involved in the progression of pediatric sepsis by regulating complement and coagulation cascades.

HCK belonged to Src family of tyrosine kinases. Its inhibitor reduced neutrophil migration and TNF-a secretion in mice and presented resistance to endotoxic shock. And it had important role in transmitting LPS signaling in macrophages $[25,26]$. In this study, HCK co-expressed with GSEC and NONHSAT160878.1 was enriched in chemokine signaling pathway. Therefore, we concluded that GSEC and NONHSAT160878.1 might be involved in the progression of pediatric sepsis by regulating chemokine signaling pathway.

NLRC4, NLR family CARD domain containing 4, and was found to be up-regulated by MAPK pathway in pediatric sepsis, which also inhibited IL-1 $\beta$ and IL-18 production to contribute to the anti-inflammatory response[27]. In this study, NLRC4 co-expressed with XR_926068.1 was enriched in NOD-like receptor signaling pathway. So XR_926068.1 might be involved in the progression of pediatric sepsis by regulating NOD-like receptor signaling pathway.

LTB4/LTB4R pathway was enriched in the pathogenesis of the sepsis[28]. Antiplatelet drugs could down regulated expression of pro-inflammatory mediators, such as cytokines and leukotriene B4 receptor (LTB4R), through modulating transcription of NF-KB and attenuated microcirculation impairment during sepsis[29]. In this study, LTB4R co-expressed with RARA-AS1 was enriched in neuroactive ligand receptor interaction. Thence, we speculated that RARA-AS1 might be involved in the progression of pediatric sepsis by regulating neuroactive ligand receptor interaction.

\section{Conclusion}

In conclusion, 8 DEMs (MAPK14, ITGAM, HK3, ALOX5, CR1, HCK, NLRC4 and LTB4R) and 4 DELs (GSEC, NONHSAT160878.1, XR_926068.1 and RARA-AS1) were identified as potential biomarkers in pediatric sepsis. Our study highlights the important roles of turquoise module in the progression of pediatric sepsis. This article comprehensively expounds the DEMs and DELs differences between pediatric sepsis and normal group, and provides more directions to study the useful diagnostic markers and molecular 
mechanism of pediatric sepsis. Several limitations still existed in this study. Large numbers of pediatric sepsis samples are needed for further researches and further experiments are required to understand the biological of key IncRNAs in pediatric sepsis.

\section{Abbreviations}

DEMs: differentially expressed mRNA; DELs: differentially expressed IncRNAs; GEO: Gene Expression Omnibus; WGCNA: weighted gene co-expression network analysis; GO: Gene Ontology; KEGG: Kyoto Encyclopedia of Genes and Genomes; IncRNAs: Long noncoding RNAs; SIRS: systemic inflammatory response syndrome; TOM: topological overlap measure; BP: biological process; MF: molecular function; CC: cellular component; MAPK14: Mitogen-activated protein kinase 14; ALI: acute lung injury; ITGAM: Integrin subunit alpha M; HK3: Hexokinase 3; LPS: lipopolysaccharide; 5-LO: 5-lipoxygenase; LTs: leukotrienes; LTB4R: leukotriene B4 receptor.

\section{Declarations}

\section{Availability of data and materials}

The datasets during the current study are available in the GEO:

https://www.ncbi.nlm.nih.gov/geo/query/acc.cgi?acc=GSE13904. Links to repositories for the annotation, visualization and integrated discovery is available in the DAVID: http://david.abcc.ncifcrf.gov/.

\section{Ethics approval and consent to participate}

Not applicable.

\section{Consent for publication}

Not applicable.

\section{Competing interests}

The authors declare that they have no competing interests.

\section{Funding}

This study was supported by Leading Talents Fund in Science and Technology Innovation in Henan Province (Grant No. 194200510017), Provincial Ministry Co-construction Project from Medical Scientific 
and Technological Research Program of Henan Province (Grant No. SBGJ2018020), Science and Technology people-benefit project of Zhengzhou (2019KJHM0001), The integrated thinking research foundation of the China foundation for International Medical Exchange (Grant No. Z-2016-23-2001), Overseas Training Program of Health Science and Technology Talents in Henan Province (HWYX2019008).

\section{Authors' Contributions}

Zhang XJ and Cui YQ participated in designing the study and drafting the manuscript. Sun TW and Zhang HB designed the study, wrote and revised the manuscript. All authors contributed to and approved the final version of the manuscript.

\section{Acknowledgements}

Not applicable.

\section{Supplementary Information}

Supplemental Fig. 1. Cluster graph of differential mRNA expression levels change in (a) control vs. pediatric sepsis day1, (b) control vs. pediatric sepsis day3, (c) control vs. pediatric septic shock day1, (d) control vs. pediatric septic shock day3, (e) control vs. pediatric SIRSday1, (f) control vs. pediatric SIRS day3 and (g) control vs. pediatric SIRS resolved day3 groups. Each column of Cluster graph represents a sample and each row represents a differential gene. Red represents a relatively high expression, and green represents a relatively low expression.

Supplemental Fig. 2. Cluster graph of differential IncRNA expression levels change in (a) control vs. pediatric sepsis day1, (b) control vs. pediatric sepsis day3, (c) control vs. pediatric septic shock day1, (d) control vs. pediatric septic shock day3, (e) control vs. pediatric SIRSday1, (f) control vs. pediatric SIRS day3 and (g) control vs. pediatric SIRS resolved day3 groups. Each column of Cluster graph represents a sample, and each row represents a differential IncRNA. Red represents a relatively high expression, and green represents a relatively low expression.

\section{References}

1. Fleischmann-Struzek C, Goldfarb DM, Schlattmann P, Schlapbach LJ, Reinhart K, Kissoon N. The global burden of paediatric and neonatal sepsis: a systematic review. Lancet Respir Med. 2018;6(3):223-30.

2. Weiss SL, Peters MJ, Alhazzani W, Agus M, Flori HR, Inwald DP, Nadel S, Schlapbach LJ, Tasker RC, Argent AC, et al. Surviving Sepsis Campaign International Guidelines for the Management of Septic 
Shock and Sepsis-Associated Organ Dysfunction in Children. Pediatr Crit Care Med. 2020;21(2):e52106.

3. Spaeder MC, Moorman JR, Tran CA, Keim-Malpass J, Zschaebitz JV, Lake DE, Clark MT. Predictive analytics in the pediatric intensive care unit for early identification of sepsis: capturing the context of age. PEDIATR RES. 2019;86(5):655-61.

4. Nagano T, Fraser P. No-nonsense functions for long noncoding RNAs. CELL.2011;145(2): 178 - 81.

5. Khorkova O, Hsiao J, Wahlestedt C. Basic biology and therapeutic implications of IncRNA. Adv Drug Deliv Rev. 2015;87:15-24.

6. Zhang TN, Li D, Xia J, Wu QJ, Wen R, Yang N, Liu CF. Non-coding RNA: a potential biomarker and therapeutic target for sepsis. Oncotarget. 2017;8(53):91765-78.

7. Dai Y, Liang Z, Li Y, Li C, Chen L. Circulating Long Noncoding RNAs as Potential Biomarkers of Sepsis: A Preliminary Study. Genet Test Mol Biomarkers. 2017;21(11):649-57.

8. Li Y, Li Y, Bai Z, Pan J, Wang J, Fang F. Identification of potential transcriptomic markers in developing pediatric sepsis: a weighted gene co-expression network analysis and a case-control validation study. J TRANSL MED. 2017;15(1):254.

9. Langfelder P, Horvath S. WGCNA: an R package for weighted correlation network analysis. BMC BIOINFORMATICS. 2008;9:559.

10. Zhang B, Horvath S. A general framework for weighted gene co-expression network analysis. Stat Appl Genet Mol Biol. 2005;4:e17.

11. Carlson MR, Zhang B, Fang Z, Mischel PS, Horvath S, Nelson SF. Gene connectivity, function, and sequence conservation: predictions from modular yeast co-expression networks. BMC GENOMICS. 2006;7:40.

12. Vachharajani $\mathrm{V}$, McCall CE. Epigenetic and metabolic programming of innate immunity in sepsis. Innate Immun. 2019;25(5):267-79.

13. Hassan Fl, Didari T, Khan F, Mojtahedzadeh M, Abdollahi M. The Role of Epigenetic Alterations Involved in Sepsis: An Overview. Curr Pharm Des. 2018;24(24):2862-69.

14. David VL, Ercisli MF, Rogobete AF, Boia ES, Horhat R, Nitu R, Diaconu MM, Pirtea L, Ciuca I, Horhat D, et al. Early Prediction of Sepsis Incidence in Critically III Patients Using Specific Genetic Polymorphisms. BIOCHEM GENET. 2017;55(3):193-203.

15. Pan W, Wei N, Xu W, Wang G, Gong F, Li N. MicroRNA-124 alleviates the lung injury in mice with septic shock through inhibiting the activation of the MAPK signaling pathway by downregulating MAPK14. INT IMMUNOPHARMACOL. 2019;76:105835.

16. Zhou H, Li Y, Gui H, Zhao H, Wu M, Li G, Li Y, Bai Z, Yin Z, Redmond HP, et al. Antagonism of Integrin CD11b Affords Protection against Endotoxin Shock and Polymicrobial Sepsis via Attenuation of HMGB1 Nucleocytoplasmic Translocation and Extracellular Release. J IMMUNOL. 2018;200(5):1771-80. 
17. Hoshi M, Osawa Y, Ito H, Ohtaki H, Ando T, Takamatsu M, Hara A, Saito K, Seishima M. Blockade of indoleamine 2,3-dioxygenase reduces mortality from peritonitis and sepsis in mice by regulating functions of CD11b + peritoneal cells. INFECT IMMUN. 2014;82(11):4487-95.

18. Sheneef A, Mohamed T, Boraey NF, Mohammed MA. Neutrophil CD11b, CD64 and Lipocalin-2: Early Diagnostic Markers of Neonatal Sepsis. Egypt J Immunol. 2017;24(1):29-36.

19. Jamsa J, Huotari V, Savolainen ER, Syrjala H, Ala-Kokko T. Kinetics of leukocyte CD11b and CD64 expression in severe sepsis and non-infectious critical care patients. Acta Anaesthesiol Scand. 2015;59(7):881-91.

20. Smith JA, Stallons LJ, Schnellmann RG. Renal cortical hexokinase and pentose phosphate pathway activation through the EGFR/Akt signaling pathway in endotoxin-induced acute kidney injury. Am J Physiol Renal Physiol. 2014;307(4):F435-44.

21. Monteiro AP, Soledade E, Pinheiro CS, Dellatorre-Teixeira L, Oliveira GP, Oliveira MG, Peters-Golden M, Rocco PR, Benjamim CF, Canetti C. Pivotal role of the 5-lipoxygenase pathway in lung injury after experimental sepsis. Am J Respir Cell Mol Biol. 2014;50(1):87-95.

22. Awwad K, Steinbrink SD, Fromel T, Lill N, Isaak J, Hafner AK, Roos J, Hofmann B, Heide H, Geisslinger $G$, et al. Electrophilic fatty acid species inhibit 5-lipoxygenase and attenuate sepsis-induced pulmonary inflammation. Antioxid Redox Signal. 2014;20(17):2667-80.

23. Brekke OL, Hellerud BC, Christiansen D, Fure H, Castellheim A, Nielsen EW, Pharo A, Lindstad JK, Bergseth G, Leslie G, et al. Neisseria meningitidis and Escherichia coli are protected from leukocyte phagocytosis by binding to erythrocyte complement receptor 1 in human blood. MOL IMMUNOL. 2011;48(15):2159-69.

24. Sakiniene E, Heyman B, Tarkowski A. Interaction with complement receptor 1 (CD35) leads to amelioration of sepsis-triggered mortality but aggravation of arthritis during Staphylococcus aureus infection. SCAND J IMMUNOL. 1999;50(3):250-5.

25. Lowell CA, Berton G. Resistance to endotoxic shock and reduced neutrophil migration in mice deficient for the Src-family kinases Hck and Fgr. Proc Natl Acad Sci USA. 1998;95(13):7580-4.

26. Leu T, Charoenfuprasert S, Yen C, Fan C, Maa M. Lipopolysaccharide-induced c-Src expression plays a role in nitric oxide and TNFalpha secretion in macrophages. MOL IMMUNOL. 2006;43(4):308.

27. Zhu G, Chen J, Tian J, Ge L, Xing A, Tang G. Expression of NLRC4 in children with septicaemia and mechanisms of NLRC4 in in vitro cytokine secretion. MOL MED REP. 2016;14(1):509-14.

28. Wilson GA, Butcher LM, Foster HR, Feber A, Roos C, Walter L, Woszczek G, Beck S, Bell CG. Humanspecific epigenetic variation in the immunological Leukotriene B4 Receptor (LTB4R/BLT1) implicated in common inflammatory diseases. GENOME MED. 2014;6(3):19.

29. Akinosoglou K, Alexopoulos D. Use of antiplatelet agents in sepsis: a glimpse into the future. THROMB RES. 2014;133(2):131-8.

\section{Figures}


a
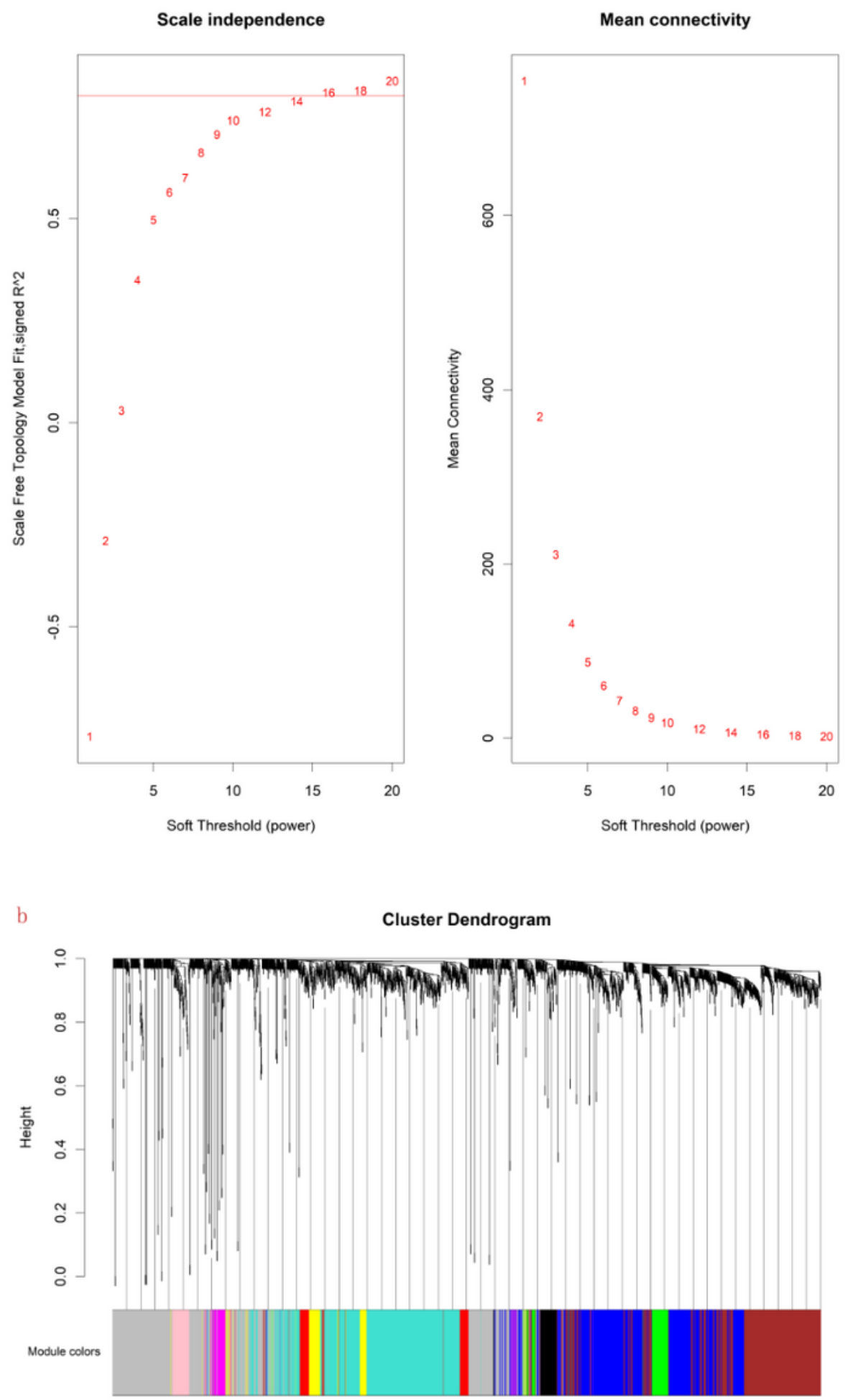

\section{Figure 1}

(a) Scale independence and mean connectivity analysis for various soft threshold powers. (b) Clustering dendrograms of DEMs. Different colors below indicates different co-expression modules. 


\section{Module-trait relationships}

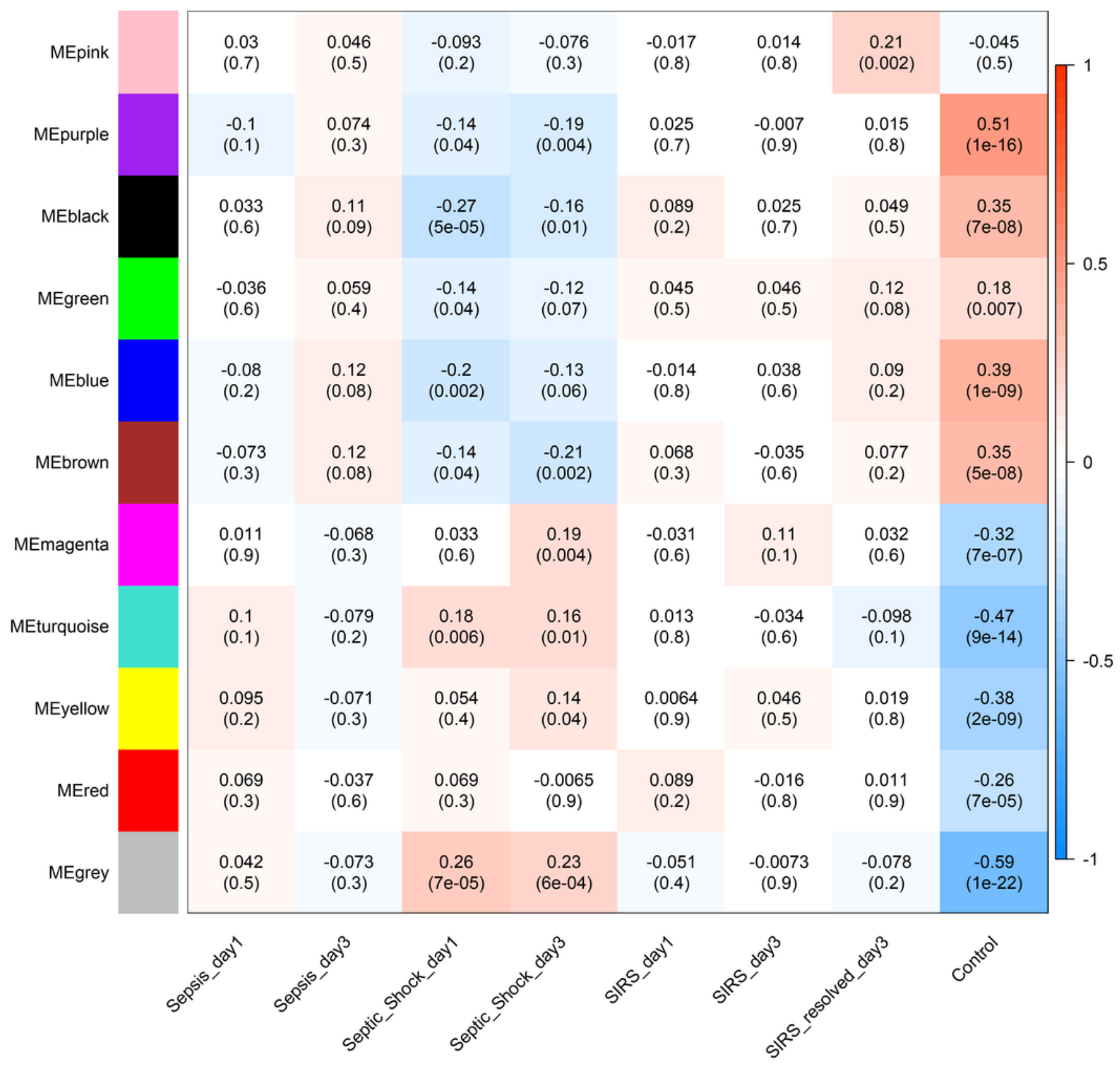

\section{Figure 2}

Module-trait relationship. Each row represents a module eigengene and each column represents a trait. Each cell includes the corresponding correlation and $p$ value. 
Module turquoise

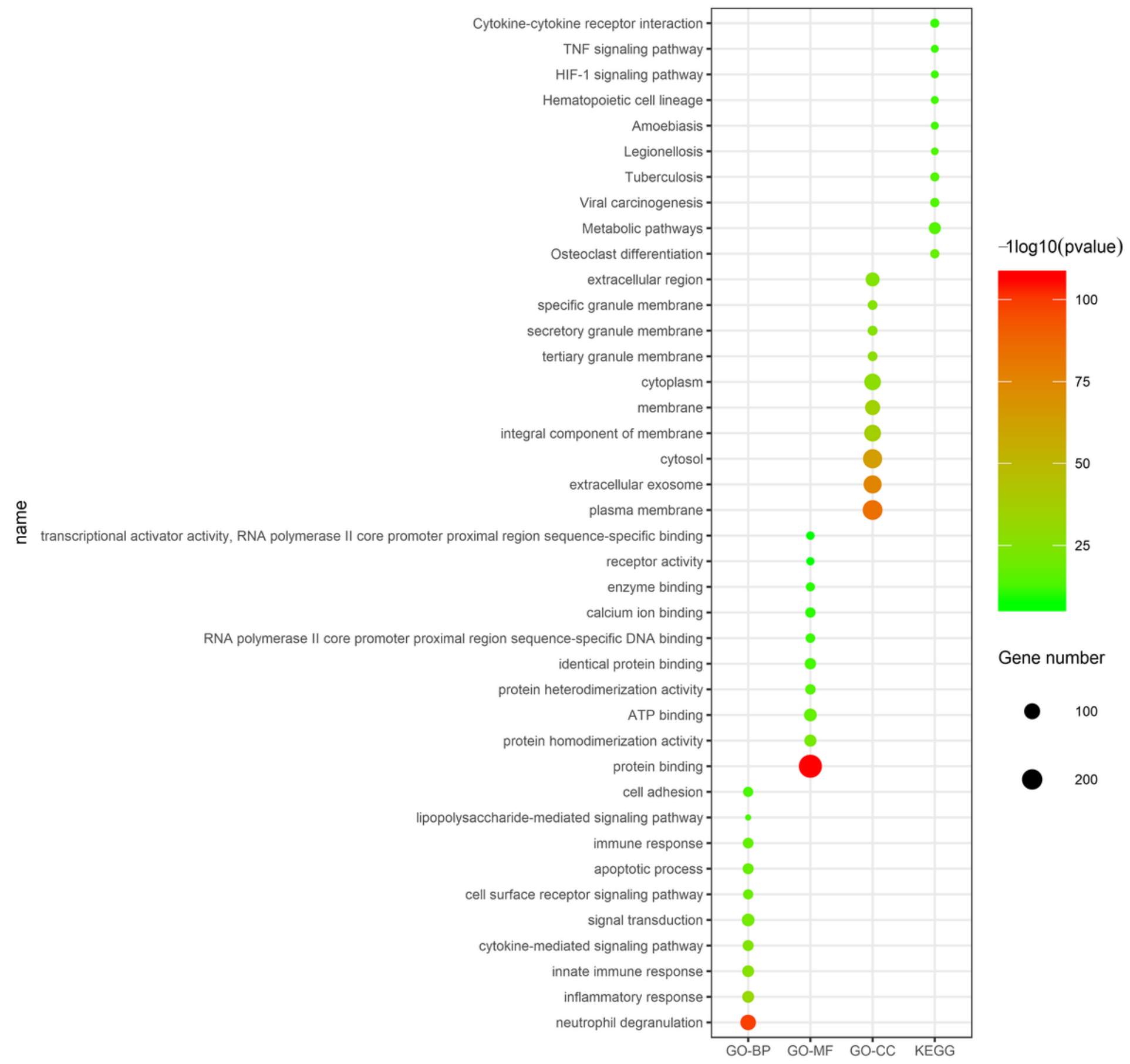

Figure 3

Enriched GO and KEGG pathways analysis of DEMs in the turquoise module. The size of spots corresponds to the numbers of DEMs, the color of spots represents $p$ value. 


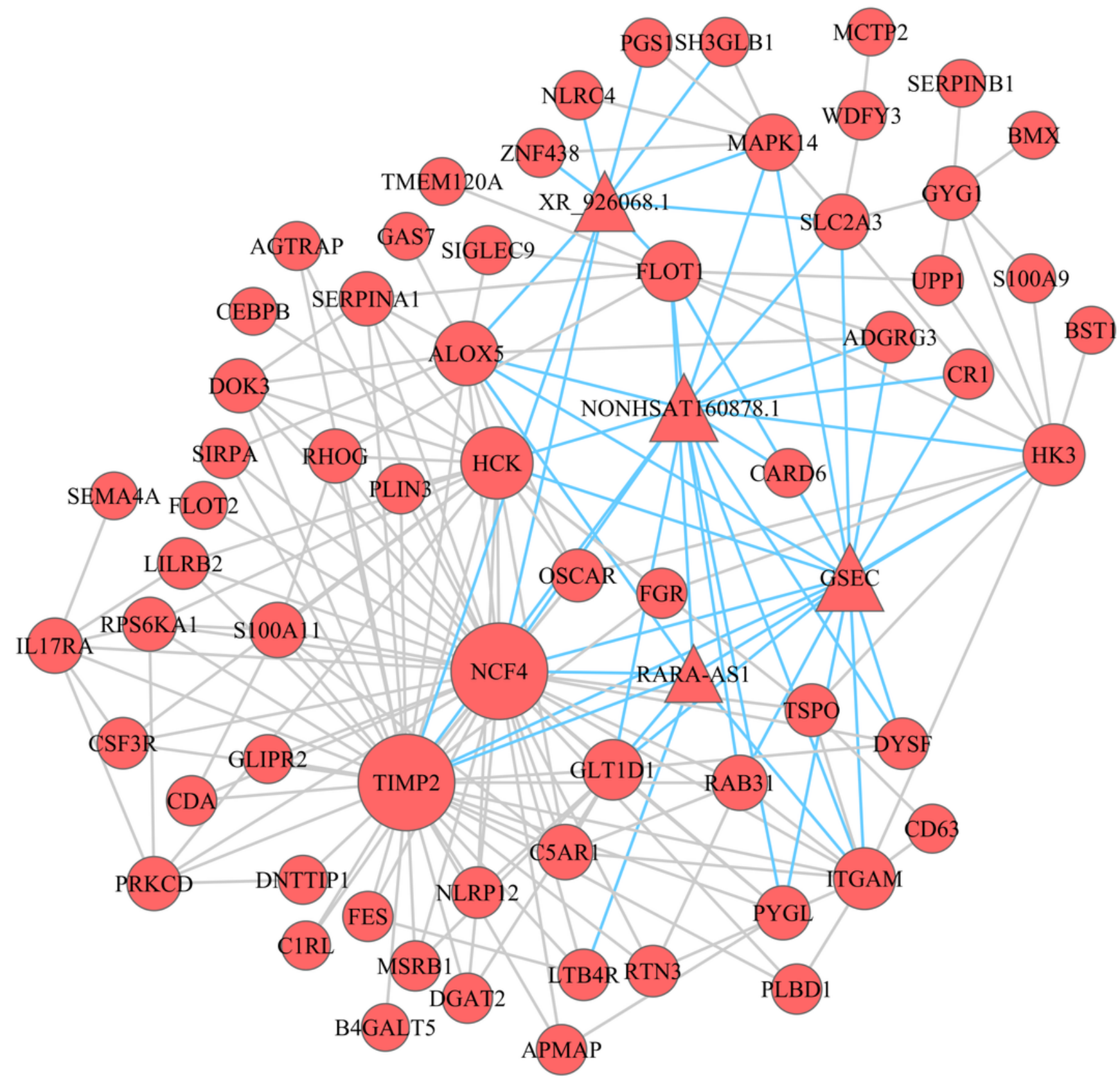

\section{Figure 4}

Co-expression DEMs-DELs network in the turquoise module. The circular nodes represent the DEMs, triangle nodes represent DELs. Blue edges represent DEMs-DELs interaction and grey edges represent DEMs- DEMs interaction. 


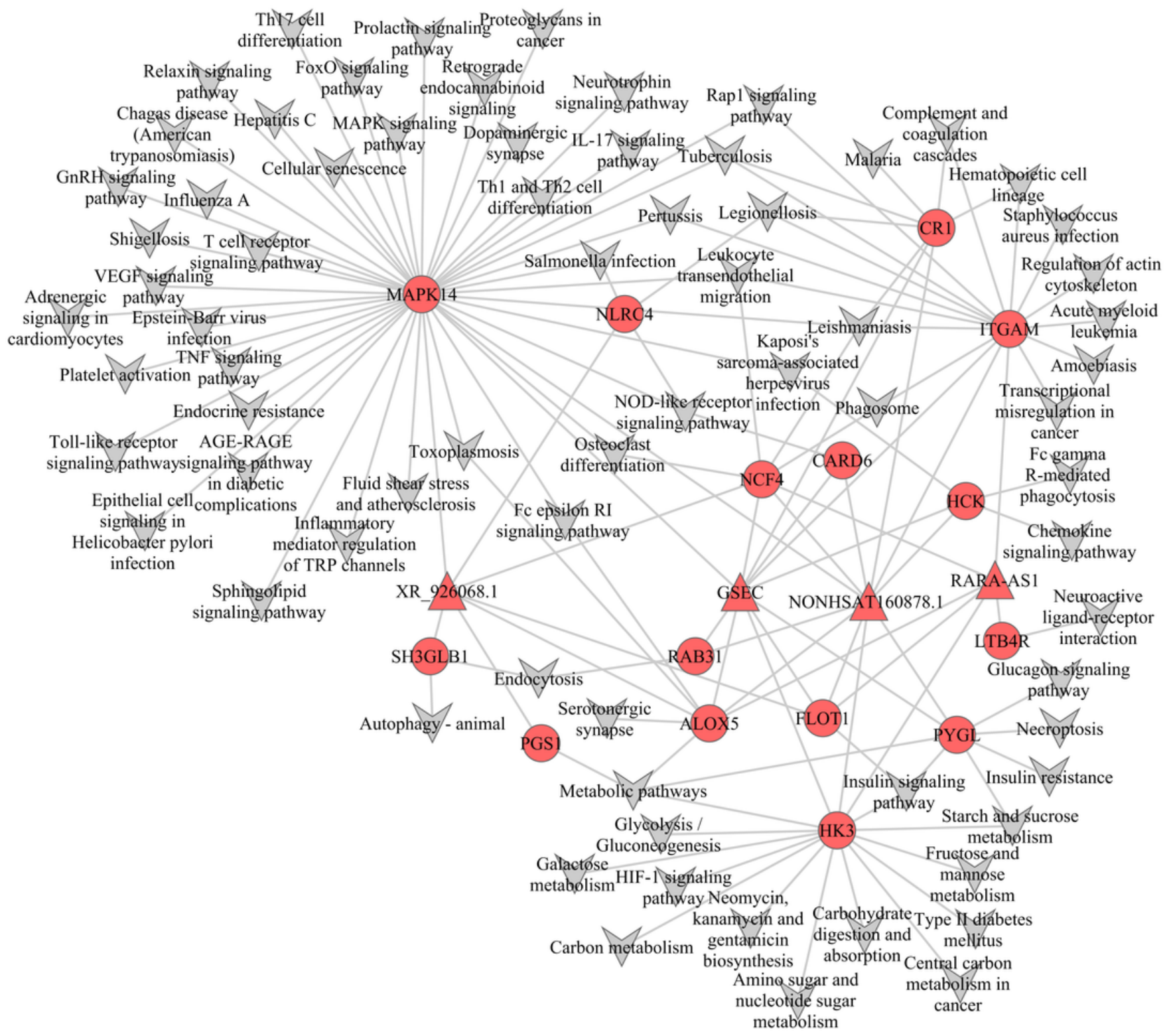

Figure 5

Co-expression DEMs-DELs pathway of the turquoise module. The circular nodes represent the DEMs, triangle nodes represent DELs.

\section{Supplementary Files}

This is a list of supplementary files associated with this preprint. Click to download.

- SupplementalFigure2.tif 
- SupplementalFigure1.tif

Page 18/18 